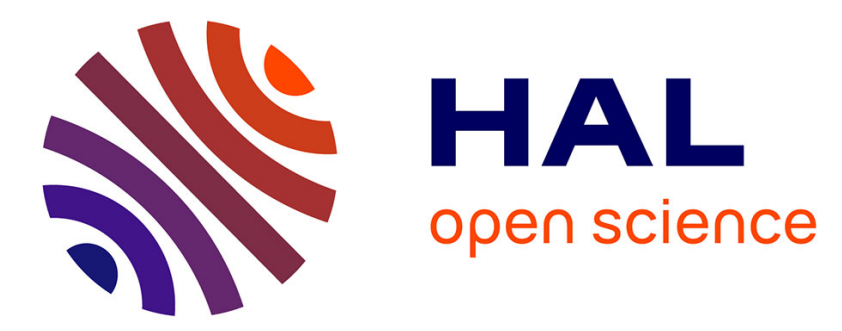

\title{
Integration of Transparent Supercapacitors and Electrodes Using Nanostructured Metallic Glass Films for Wirelessly Rechargeable, Skin Heat Patches
} Sangil Lee, Sang-Woo Kim, Matteo Ghidelli, Hyeon Seok An, Jiuk Jang, Andrea Li Bassi, Sang-Young Lee, Jang-Ung Park

\section{To cite this version:}

Sangil Lee, Sang-Woo Kim, Matteo Ghidelli, Hyeon Seok An, Jiuk Jang, et al.. Integration of Transparent Supercapacitors and Electrodes Using Nanostructured Metallic Glass Films for Wirelessly Rechargeable, Skin Heat Patches. Nano Letters, 2020, 20 (7), pp.4872-4881. 10.1021/acs.nanolett.0c00869 . hal-03247075

\section{HAL Id: hal-03247075 \\ https://hal.science/hal-03247075}

Submitted on 2 Jun 2021

HAL is a multi-disciplinary open access archive for the deposit and dissemination of scientific research documents, whether they are published or not. The documents may come from teaching and research institutions in France or abroad, or from public or private research centers.
L'archive ouverte pluridisciplinaire HAL, est destinée au dépôt et à la diffusion de documents scientifiques de niveau recherche, publiés ou non, émanant des établissements d'enseignement et de recherche français ou étrangers, des laboratoires publics ou privés. 


\section{Integration of Transparent Supercapacitors and Electrodes Using Nanostructured Metallic Glass Films for Wirelessly Rechargeable, Skin Heat Patches}

Sangil Lee, Sang-Woo Kim, Matteo Ghidelli, Hyeon Seok An, Jiuk Jang, Andrea Li Bassi, Sang-Young Lee, and Jang-Ung Park

Nano Lett., Just Accepted Manuscript • DOI: 10.1021/acs.nanolett.0c00869 • Publication Date (Web): 04 May 2020

Downloaded from pubs.acs.org on May 4, 2020

\section{Just Accepted}

"Just Accepted" manuscripts have been peer-reviewed and accepted for publication. They are posted online prior to technical editing, formatting for publication and author proofing. The American Chemical Society provides "Just Accepted" as a service to the research community to expedite the dissemination of scientific material as soon as possible after acceptance. "Just Accepted" manuscripts appear in full in PDF format accompanied by an HTML abstract. "Just Accepted" manuscripts have been fully peer reviewed, but should not be considered the official version of record. They are citable by the Digital Object Identifier (DOI®). "Just Accepted" is an optional service offered to authors. Therefore, the "Just Accepted" Web site may not include all articles that will be published in the journal. After a manuscript is technically edited and formatted, it will be removed from the "Just Accepted" Web site and published as an ASAP article. Note that technical editing may introduce minor changes to the manuscript text and/or graphics which could affect content, and all legal disclaimers and ethical guidelines that apply to the journal pertain. ACS cannot be held responsible for errors or consequences arising from the use of information contained in these "Just Accepted" manuscripts. 


\title{
Integration of Transparent Supercapacitors and
}

\section{Electrodes Using Nanostructured Metallic Glass}

\section{Films for Wirelessly Rechargeable, Skin Heat}

\section{Patches}

\author{
Sangil Lee ${ }^{1,2 \dagger}$, Sang-Woo Kim ${ }^{3 \dagger}$, Matteo Ghidellit, ${ }^{4 \dagger}$, Hyeon Seok An ${ }^{1,2 \dagger}$, Jiuk Jang ${ }^{1,2 \dagger}$, \\ Andrea Li Bassi ${ }^{4 *}$, Sang-Young Lee ${ }^{3 *}$ and Jang-Ung Park ${ }^{1,2 *}$
}

${ }^{1}$ Nano Science Technology Institute, Department of Materials Science and Engineering, Yonsei University, Seoul, 03722, Republic of Korea

${ }^{2}$ Center for Nanomedicine, Institute for Basic Science (IBS), Seoul, 03722, Republic of Korea ${ }^{3}$ Department of Energy Engineering, School of Energy and Chemical Engineering, Ulsan National Institute of Science and Technology (UNIST), Ulsan, 44919, Republic of Korea

${ }^{4}$ Dipartimento di Energia, Laboratorio Materiali Micro e Nanostrutturati, Politecnico di Milano, via Ponzio 34/3, I-20133 Milano, Italy

${ }^{5}$ Laboratoire des Sciences des Procédés et des Matériaux (LSPM), CNRS, Université Sorbonne Paris Nord, 93430, Villetaneuse, France

$\dagger$ These authors contributed equally to this work.

*Corresponding author. E-mail: jang-ung@yonsei.ac.kr; syleek@unist.ac.kr; andrea.libassi@polimi.it 


\section{ABSTRACT}

Here we demonstrate an unconventional fabrication of highly transparent supercapacitors and electrodes using random networks of nanostructured metallic glass nanotroughs for their integrations as wirelessly rechargeable and invisible, skin heat patches. Transparent supercapacitors with fine conductive patterns were printed using an electrohydrodynamic jet-printing. Also, transparent and stretchable electrodes, for wireless antennas, heaters and interconnects, were formed using random network based on nanostructured $\mathrm{CuZr}$ nanotroughs and $\mathrm{Ag}$ nanowires with superb optoelectronic properties (sheet resistance of $3.0 \Omega / \mathrm{sq}$ at transmittance of $91.1 \%$ ). Their full integrations, as an invisible heat patch on skin, enabled the wireless recharge of supercapacitors and the functions of heaters for thermal therapy of skin tissue. The demonstration of this transparent thermotherapy patch to control the blood perfusion level and hydration rate of skin suggests a promising strategy toward next-generation wearable electronics.

\section{KEYWORDS}

Nanostructured metallic glasses, transparent electrodes, transparent supercapacitors, wearable electronics, stretchable electronics 


\section{INTRODUCTION}

Recent advances in skin-attachable devices have driven considerable attention in broad fields such as displays ${ }^{1,2}$, motion detection sensors ${ }^{3-5}$, drug delivery devices ${ }^{6}$, and biosensors ${ }^{7-}$ 10. Most studies have been mainly focused on developing flexible and stretchable skinattachable devices for the comfort of individuals ${ }^{11,12}$. However, the transparency of the skinattachable devices is one of the most important factors for user's aesthetic satisfaction. In addition to the transparency, wireless operation is another crucial factor for skin-attachable devices to avoid interfering with the user's routine activity. Nonetheless, most skin-attachable devices are not transparent ${ }^{11,13}$ or only partially transparent ${ }^{12,14-16}$, and they often require additional bulky devices for the wireless operation such as modules, power sources (i.e. batteries) ${ }^{13,17-20}$. Even though it is important to minimize the power sources, there are limitations for such skin-attachable devices to apply conventional power sources. The transparent power sources with reliable electrochemical performance should be indispensably required to operate the device successfully. Thus, several requisites must be considered for the next-step skin-attachable devices. These requisites include 1) high transparency and flexibility over all components, 2) an independent energy device that supplies consistent operating power to the functional components, 3) the wireless power transmission without additional wiring for power supply.

Herein, we fabricated a wirelessly rechargeable, transparent and flexible heat patch (dimension: $1 \times 1 \mathrm{~cm}^{2}$ ) to demonstrate an example of the skin-attachable device that satisfied requisites mentioned above. To the best of our knowledge, this is the first practical demonstration that presents all components integrated within the skin-attachable device, including antenna, wireless power transmission circuit, and energy storage device, to be transparent and flexible. To fabricate a transparent and flexible device, metallic glass 
nanotroughs (NTs) and Ag nanowires (AgNWs) were hybridized ${ }^{19,21-24}$. This CuZr NTsAgNWs hybrid electrode shows low sheet resistance $\left(R_{S}\right)(3.0 \Omega / \mathrm{sq})$ and high transmittance $(T)$ (91.1\% in visible light range). Also, the electrical stability under the mechanical deformation was verified. Especially, high elasticity of metallic glass can provide better mechanical stability against deformations, which is important to reduce the damage of wearable electronic devices caused by human activity ${ }^{19}$. Using this hybrid electrode, the transparent antenna with the rectifier circuit was fabricated to develop a transparent and flexible wireless power transmission circuit. In addition, we fabricated a new transparent supercapacitor (SC) with exceptional high-resolution via an electrohydrodynamic (EHD) jet-printing technique. The energy transferred wirelessly through the power transmission circuit charges SC, and this charged energy is emitted through the antenna as heat when thermal therapy is required. By integrating this transparent and flexible SC into the circuits, we successfully produced a wireless skin-attachable device by fabricating all the components in a transparent and flexible form. Furthermore, the increased blood perfusion and the hydration rate of the skin through thermal therapy using the device provide the potential of the heat patch as a healthcare device.

\section{RESULTS}

To fabricate transparent electrode, we exploit the potential of pulsed laser deposition (PLD) to synthesize nanostructured thin film metallic glasses (TFMGs). CuZr NTs networks were prepared by electrospinning and PLD process. Polyvinylpyrrolidone (PVP) was electrospun first to form a free-standing nanofiber web on a metal collector ${ }^{19,25,26}$. More details about the fabrication of CuZr metallic glasses NTs are described in Supporting Information. Nanostructured $\mathrm{Cu}_{50} \mathrm{Zr}_{50}$ (\% at.) TFMGs have been deposited on a nanofiber web by PLD, enabling a combined control of film stoichiometry and morphology ${ }^{27}$. Figure 1a shows a schematic of the PLD process in which high energy $(850 \mathrm{~mJ})$ ns-pulsed laser hits a target whose 
ablated species (i.e. $\mathrm{Cu}-\mathrm{Zr}$ ions, atoms, and clusters) are then condensed on a substrate (i.e. NT network), see Supporting Information for more details. In order to develop a transparent electrode, thin $(\sim 120 \mathrm{~nm})$ CuZr metallic glass films have been deposited. Such low thickness enables the activation of mechanical size effects occurring at the sub-micrometer scale for TFMGs, promoting large ductility and fracture strength ${ }^{28,29}$. In addition, TFMGs have shown their potential as biocompatible coatings, while reporting high corrosion resistance and metallic conductivity $^{30,31}$. Figure $\mathrm{S} 1$ shows the scanning electron microscopy (SR) image of the $\mathrm{CuZr}$ NTs with its thin, convex, and half-shell structure. Figure $1 \mathrm{~b}$ shows that CuZr TFMGs deposited on NT are characterized by cluster-assembled growth, low density and significant porosity. Further details about morphology and structure by SEM cross-section and X-ray diffraction (XRD) are reported in Figure S2. As shown in Figure 1c, the resulting network with $\mathrm{CuZr}$ deposited on the free-standing PVP fibers can be transferred onto various desirable substrates. After the successive removal of the sacrificial PVP template-by dissolving it in acetone, isopropyl alcohol (IPA), and deionized (DI) water, AgNWs were electrosprayed to promote the connection among CuZr NTs and guarantee electron percolation (Figure 1c). The final structure results in a CuZr NTs-AgNWs hybrid network (Figure 1d) (see Supporting Information).

Figure 1e shows the relationship between the $R_{s}$ and $T$ for the nanoporous CuZr NTs network and CuZr NTs-AgNWs hybrid electrode indicating the trade-off between electrical and optical properties. From Figure 1e, it can also be noted that AgNWs don't significantly affect the $R_{s}$ (which is only slightly reduced) while guaranteeing a better electron percolation and mechanical cohesion. In addition, nanoporous $\mathrm{CuZr}$ NTs are characterized by high transparency which can be larger than $90 \%$ for samples deposited on low density fibers (electrospinnig time $<10 \mathrm{sec}$ ), as also reported in our previous study for CuZr NTs deposited by the sputtering method ${ }^{19}$. Figure $1 \mathrm{f}$ reports relative changes in the $R_{S}$ of CuZr NTs electrodes 
as a function of the radius of curvature corresponding to bending induced strain. No significant change in $R_{S}$ was observed even when the electrodes were bent to a radius of curvatures as small as $2 \mathrm{~mm}$ indicating a superior combination of conductivity and flexibility. Finally, Figure $1 \mathrm{~g}$ shows that low $R_{s}(<4 \Omega / \mathrm{sq}$ ) is kept even if large tensile strain (up to 70\%) was applied. This implies that the CuZr NTs-AgNWs hybrid network does not break while keeping good electron percolation. This high stretchability without failure indicates good adhesion at the network joints, with the combined contribution of the activation of mechanical size effects for sub-micrometer scale $\mathrm{TFMGs}^{29}$, with the presence of the large density of glass-glass interfaces and free volume as provided by the PLD microstructure. This prevents the development of shear bands while leading to high stretchability ${ }^{32}$. In our approach, transparent and stretchable electrodes for this antenna were demonstrated using a random network of CuZr NTs-AgNWs hybrid structure.

Figure 2a shows an antenna with a 5-turns structure (antenna width of $800 \mu \mathrm{m}$, turn space of $100 \mu \mathrm{m}$ ) fabricated using this hybrid electrode made of a random network of AgNWs sprayed on top of the CuZr NTs network. As a transparent antenna, the CuZr NTs-AgNWs network formed by electrospinning of CuZr NTs for 10 seconds with the spraying of AgNWs for 60 seconds had a significantly low $R_{s}$ of $3.0 \Omega /$ sq with a $T$ of $91.1 \%$ (Figure $2 \mathrm{~b}$ ). These sprayed AgNWs could bridge across the locally disconnected, open areas of the CuZr NTs network to preserve the resistance of these narrow antenna patterns (Figure S3). This CuZr NTs -AgNWs hybrid structure could be patterned photolithographically to form the antenna using dry etching. To compare resonance characteristics of the hybrid antenna with a conventional $\mathrm{Cu}$ antenna, we fabricated the $\mathrm{Cu}$ antenna as the same structure with the hybrid antenna (Figure S4). As shown in Figure 2c, both antennas showed the same resonance frequencies (13.6 MHz), and similar values of the minimum reflection (hybrid antenna: -64, Cu antenna: -69). A quality 
(Q) factor means the frequency selectivity to a given frequency. The hybrid antenna had high Q factors of $\sim 45.5$ for the transmission distance of $10 \mathrm{~mm}$. Compare to the brittle $\mathrm{Cu}$ antenna which has the same dimension, this hybrid antenna exhibited good mechanical stretchability with a negligible change in its resistance $\left(\Delta R / R_{0}<1.5 \%\right)$ under a tensile strain of $30 \%$, as shown in Figure 2d. For example, the $\mathrm{Cu}$ antenna showed mechanical failure at a tensile strain of $10 \%$, contrast to the negligible change in a $R_{s}$ of the hybrid antenna (Figure S5). In addition, this hybrid antenna can be used as a flexible and invisible heater. Figure $2 \mathrm{e}$ shows the temperature as a function of time with $0.9 \mathrm{~V}$ direct current (DC) bias. By applying constant DC bias, the temperature was raised and then saturated to the desired value within 9 seconds. Furthermore, this hybrid heater exhibits outstanding flexibility, as shown in the photo and infrared (IR) image in Figure 2f, and in the graph in Figure S6. The heater bent with a $20 \mathrm{~mm}$ radius of curvature while maintaining the DC bias $(0.9 \mathrm{~V})$. This bending did not change the temperature significantly $\left(\Delta T / T_{0} \sim 2.3 \%\right)$, as shown in Figure S6.

EHD jet-printing, a state-of-the-art drop-on-demand printing technique, has recently been spotlighted as a high-fidelity/large-area patterning technique in various microscale electronic devices ${ }^{33,34}$. We fabricate a new transparent flexible SC with exceptional highresolution via an EHD jet-printing technique (Figure S7). A stepwise fabrication procedure of the transparent and flexible SC is schematically illustrated in Figure 3a. Details about the fabrication procedure of SC devices are provided in Supporting Information. Figure 3b shows the structural features of the microscale grid-patterned SC electrodes. Conventional inkjet printing techniques have limitations in lowering widths of printed Ag grids (mostly, > 35 $\mu \mathrm{m})^{35,36}$. By sharp contrast, the EHD jet-printed Ag grids enabled a significant reduction in the line width and height (Figure S8). Notably, the line width is far smaller than those directly detectable by human eyes, demonstrating a viable role of the EHD jet-printing in realizing the 
fine-resolution images that are essentially needed for transparent devices ${ }^{33,34,37}$. Moreover, the grid pattern in the SC electrode was not structurally disrupted after spin coating-assisted deposition of the PEDOT:PSS layer on top of the Ag grids. The uniform distribution of the PEDOT:PSS was verified by tracing sulfur (S) elements in an energy-dispersive X-ray spectroscopy (EDS) mapping image (inset of Figure 3b). Figure 3c presents the electrical/optical properties of the above-prepared SC electrodes as a function of Ag grid pitch. In overall, the $T$ of the SC electrodes tended to improve with increasing the grid pitch, whereas their $R_{S}$ became larger. Such a trade-off between the $T$ and $R_{S}$ is ascribed to the scattering and reflection of incident light on the $\mathrm{Ag}$ grid patterns ${ }^{34,37,38}$. Notably, over a whole range of the grid pitches examined herein, the $T$ of the SC electrodes remained higher than $70 \%$ (Figure S9). Meanwhile, the $R_{s}$ of the SC electrodes increased from $0.4 \Omega / \mathrm{sq}(T$ of $72 \%)$ to $2.3 \Omega / \mathrm{sq}(T$ of $93 \%$ ). These results demonstrate a promising potential of the EHD jet-printed SC electrodes for transparent device applications. The resulting SC device showed a $T$ above $78 \%$ in the visible range (Figure 3d). Although the $T$ of the SC device was lower than that of the SC electrode, it was high enough to detect the images beneath the device (inset of Figure 3d).

To successfully apply a transparent SC device to flexible thermotherapy patch, its thermal stability should be secured as a prerequisite. We monitored the change in the $T$ of the transparent SC device as a function of temperatures (Figure 3e). The $T$ remained almost unchanged over a wide range of temperature. Moreover, the transparent SC device showed good capacitance retention up to 2,000 cycles at a high temperature of $40{ }^{\circ} \mathrm{C}$ (Figure S10). We investigated the electrochemical performance of the transparent SC device. The cyclic voltammetry $(\mathrm{CV})$ curves presented normal pseudo-capacitive charge/discharge behavior (Figure 3f), which appeared similar to those of conventional pseudo-capacitors based on conducting polymers. In addition, the transparent SC device showed normal symmetric 
galvanostatic charge/discharge (GCD) profiles at various current densities of 5 to $30 \mu \mathrm{A} \mathrm{cm}{ }^{-2}$ (inset of Figure $3 \mathrm{~g}$ ). The cycling stability of the transparent SC device was examined at a constant charge/discharge current density of $50 \mu \mathrm{A} \mathrm{cm}^{-2}$ (Figure $3 \mathrm{~g}$ ). After 5,000 cycles, the specific capacitance was maintained above $90 \%$ of the initial value. This superb cycling performance, in collaboration with a wireless charging system, is expected to enable long-term use of the integrated transparent heaters. We quantitatively investigated the mechanical flexibility of the transparent SC device. After 1,000 bending cycles, the SC device still retained their structural integrity without impairing the cell capacitance (Figure 3h). Even upon being subjected to bending deformation (the bending radii were varied from 20 to $4 \mathrm{~mm}$ ), the $\mathrm{CV}$ curves of the transparent SC device remained almost unchanged (Figure S11). This result indicates that the transparent SC device presented herein can be easily integrated with various flexible/wearable electronics.

The wireless operation is mandatory functionality to enhance the user experience of wearable electronics, especially for skin-attachable devices ${ }^{39}$. Figure 4 discusses the fabrication process, electrical properties, and operating mechanisms of a transparent heat patch integrated with the circuits for wireless functionalities. The device consists of three electrical components (rectifier, antenna, and SC) as shown in Figure 4a. A transparent heat patch was fabricated by integrating the antenna, rectifier circuits, and SC (Figure 4b). Figure S12 shows a photograph of the heat patch before the integration with the energy storage system. Details about the fabrication of the heat patch are described in Figure S13 and Methods. All components of the device except the Si diode were manufactured using the transparent materials to maximize the $T$. As shown in Figure S14, the heat patch shows outstanding $T$ ( $\sim 72 \%$ in visible light range) even after incorporating all components. Figure $4 \mathrm{c}$ shows the rectifying characteristics of the wireless power transfer unit including the antenna and rectifier 
elements. The rectifier circuit was composed of the p-type/intrinsic/n-type (PIN) diodes fabricated using an ultrathin $\mathrm{Si}$ nanomembrane (thickness: $200 \mathrm{~nm}$ ) and $\mathrm{SiO}_{2}$-based capacitor (Figure S15). In this circuit, the antenna wirelessly receives power inductively (black line in Figure $4 \mathrm{c}$ ). The rectifier circuit converts the alternating current (AC) bias (red line in Figure 4c) of 5.2 peak to peak voltage $\left(\mathrm{V}_{\mathrm{pp}}\right)$ at $13.6 \mathrm{MHz}$ into DC bias (blue line in Figure 4c) of 2.01 root mean square voltage $\left(\mathrm{V}_{\mathrm{rms}}\right)$.

Figure $4 \mathrm{~d}$ shows circuit diagrams of the heat patch and the mechanisms during the wireless operation. Since the switch is opened during the charging process, the wirelessly applied AC power is rectified to DC power along the rectifier circuit to charge the SC. In contrast, the switch is closed during the discharging process, and the power from the SC is applied directly to the antenna to generate heat. Figure $4 \mathrm{e}$ shows the results of the chargedischarge test. During the 30 cycles of switching on and off test, there was negligible degradation of maximum charging voltage and it maintained about $0.95 \mathrm{~V}$. The rectified DC power $\left(2.01 \mathrm{~V}_{\text {rms }}\right)$ was also sufficient to charge the SC. Figure $4 \mathrm{f}$ shows the operating mechanisms of the switch when external pressure is applied. As shown in the schematic image, the power transmission circuit and the SC are connected by silver paste, and the switch is not connected to the electrode physically unless pressure is applied. When pressure is applied to the switch, the switch is connected to the electrode of the SC to heat the antenna using the stored energy of the SC. The physical contact between these electrodes can turn on this switch to operate the heat patch. In order to measure the minimum tactile pressure that can turn on the switch, we applied the tactile pressure with a gradual increment on the device using a commercial, programmable compression test instrument (Mark-10, Mark-10 Corp., USA). As plotted in Figure S16, the minimal tactile pressure of $18 \mathrm{kPa}$ can turn on the switch, which is similar to the pressure level for gentle finger touch. The heat patch was attached to the back of 
the hand to demonstrate the skin-attachable device (Figure 4g). As shown in the photograph, the heat patch attached to the human skin is inconspicuous due to the superb $T$. The heat patch can receive inductively coupled AC power wirelessly from a transmitting coil (at $13.6 \mathrm{MHz}$ ) at the distance of $10 \mathrm{~mm}$. More details about wireless power transmission of the heat patch are described in Supporting Information. Figure 4h shows IR images before and after the switch is pressed. When the switch was pressed, the heat patch started to generate heat. Subsequently, the temperature of the heater was increased and then saturated to target temperature within 3 seconds (Supporting Movie S2). All the on/off tests were performed with one charge, and three tests showed a constant temperature about $40.6{ }^{\circ} \mathrm{C}$ (Figure $4 \mathrm{i}$ ). Therefore, there is no concern about low-temperature burns $\left(>45^{\circ} \mathrm{C}\right)$ due to the heater.

Thermal therapy is one of the most popular physiotherapy methods, and it is effective to increase blood circulation, reduce inflammation and pain ${ }^{40,41}$. Also, thermal therapy enhances transdermal molecular movement, thus increasing the skin permeability of active material ${ }^{42}$. Here, we measured blood perfusion and skin hydration rate to demonstrate the effectiveness of the heat patch. First, the increase in blood flow due to capillary dilation that is one of the effects of thermal therapy was measured by means of a laser Doppler imaging (LDI) device $^{43}$. Figure 5a presents a schematic image of the blood flow measurement steps before and after thermal therapy. After thermal therapy $\left(41^{\circ} \mathrm{C}\right)$ to a local skin area (back of a hand, dimension: $1 \times 1 \mathrm{~cm}$ ) for about 1 minute with the manufactured heat patch, the local blood perfusion was measured by LDI. Immediately after thermal therapy, the blood flow peaked up and then gradually decreased over time. It was found that a short-lasting heat application (41 ${ }^{\circ} \mathrm{C}$ for $60 \mathrm{sec}$ ) causes significant cutaneous hyperemia existing for up to 13 minutes (Figures $5 \mathrm{~b}, \mathrm{~S} 17)$. Figure $5 \mathrm{c}$ shows the results for laser Doppler perfusion monitoring at one spot of skin. Measurements are expressed as perfusion units (PU), which are arbitrary ${ }^{44}$. Compared to 
before thermal therapy, the skin heated for 1 minute $\left(41^{\circ} \mathrm{C}\right)$ presented 3.8 times more blood flow rate than normal skin.

We also measured the increase in transdermal absorption of active material to prove the effectiveness of thermal therapy. Figure $5 \mathrm{~d}$ shows a schematic image of the mechanisms for the skin moisture changes measurement. More details about measurement of skin moisture changes are described in Supporting Information. Figure 5e presents the average of the hydration state of the skin when the heat is applied by the heat patch for $0,10,40$, and 60 seconds immediately after applying moisture cream. More details of the experimental data are shown in Figure S18. The results show that the hydration states (arbitrary unit, a.u.) of the skin without moisture cream applied and the skin with only moisture cream applied were 32.3 and 47.6. After the application of moisture cream, the hydration state (a.u.) after 10, 40 and 60 seconds of thermal therapy was $69.7,84.1$ and 90.7 . As thermal therapy time increased from 10 seconds to 60 seconds, the absorption rate of moisture cream increased linearly. In addition, the moisture absorption rate of the skin after thermal therapy was increased by 1.9 times when the hydration rate of the skin without thermal therapy (47.6) and the skin hydration rate (90.7) after 60 seconds of thermal therapy were compared. Our data showed that the best combination (heat temperature and time) was $41{ }^{\circ} \mathrm{C}$ for $60 \mathrm{sec}$ that could remarkably exalt the skin hydration rate compared with other times.

\section{CONCLUSION}

In summary, this work presented the advantages of heat patches with transparent, flexible, wireless, rechargeable characteristics. The antenna in the heat patch for wireless data transmission using $\mathrm{CuZr}$ NTs-AgNWs hybrid network presents superb optoelectronic properties $\left(R_{s}\right.$ of $3.0 \Omega / \mathrm{sq}$ at $T$ of $91.1 \%$ ) and mechanical robustness against folding and stretching deformation. The fabricated antenna wirelessly received AC power from the reader 
coil, and the rectifier circuit converted AC power to DC power. In addition, as an energy storage device, the transparent and flexible SC was integrated with the power transmission circuit. We successfully produced a heat patch as an example of the skin-attachable device and demonstrated the possibility of thermal therapy by measuring blood flow and skin hydration. We believe our approach presents the substantial promise of future skin-attachable electronics. 


\section{FIGURES}

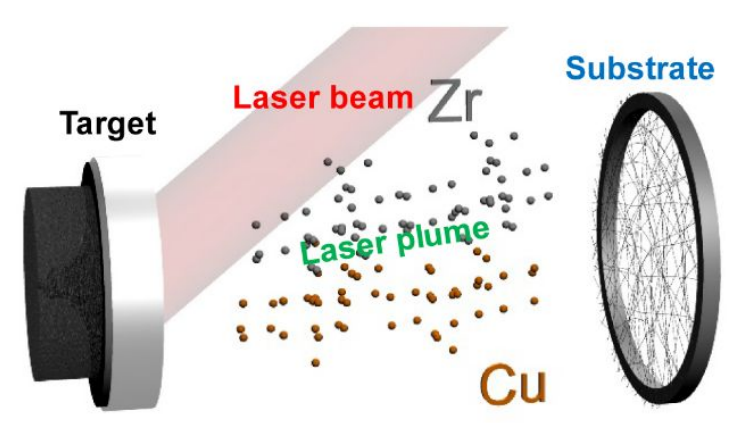

c
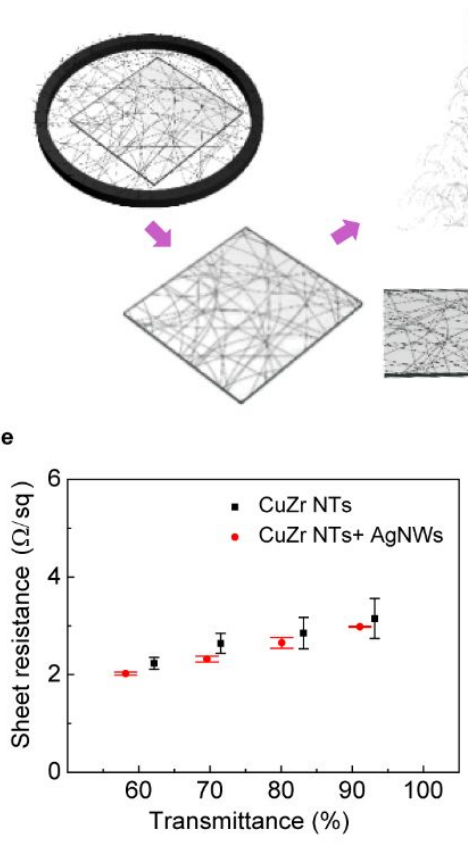

b

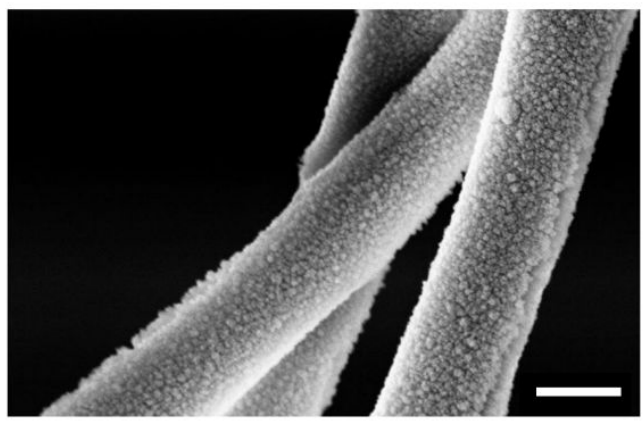

d

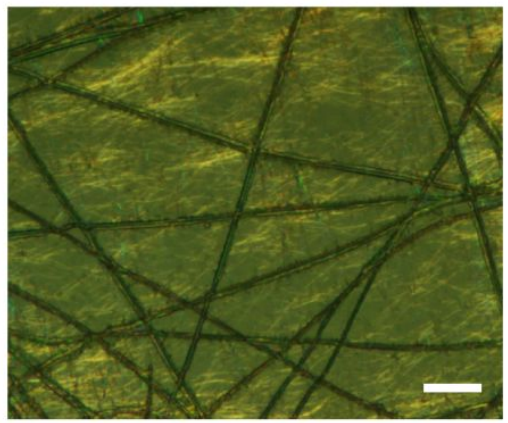

g

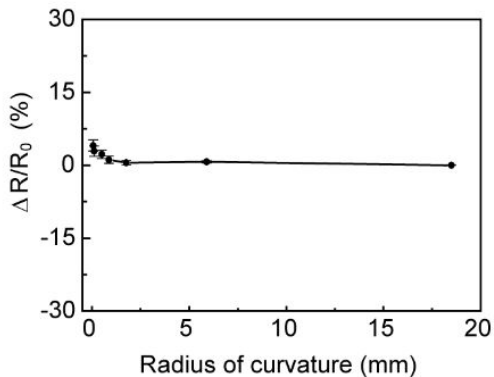

Figure 1. Metallic glass nanotroughs (a) Schematic illustration of the pulsed laser deposition process of the CuZr NTs network. (b) SEM image of a CuZr NTs network. Scale bar, $1 \mu \mathrm{m}$. (c) Schematic illustration of the fabrication process of CuZr NTs-AgNWs hybrid network. (d) Optical microscope image of the CuZr NTs-AgNWs hybrid structure. Scale bar, $20 \mu \mathrm{m}$. (e) Transmittance versus sheet resistance of the CuZr NTs and CuZr NTs-AgNWs hybrid electrode. (f) Relative resistance changes as a function of the bending radius of the CuZr NTs and CuZr NTs-AgNWs hybrid electrode. (g) Sheet resistance of the CuZr NTs and CuZr NTsAgNWs hybrid electrode as a function of tensile strain. 
d

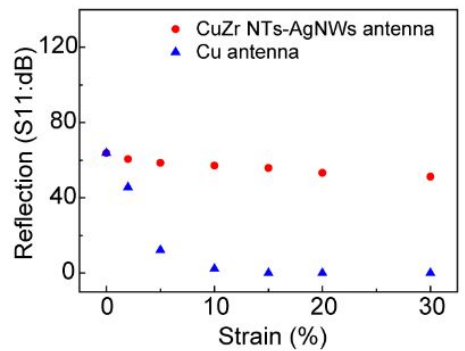

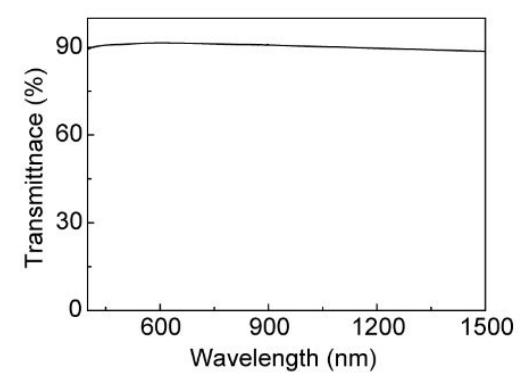

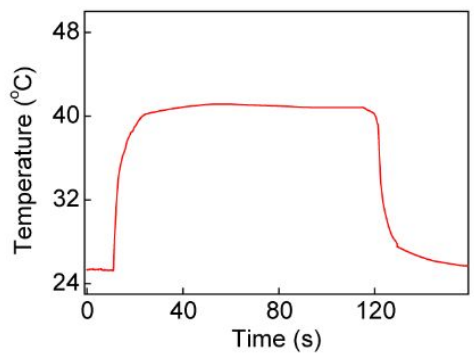

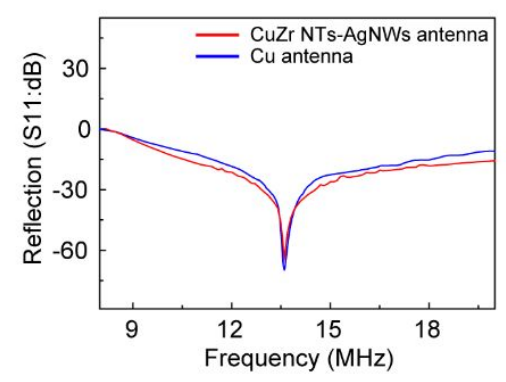

f

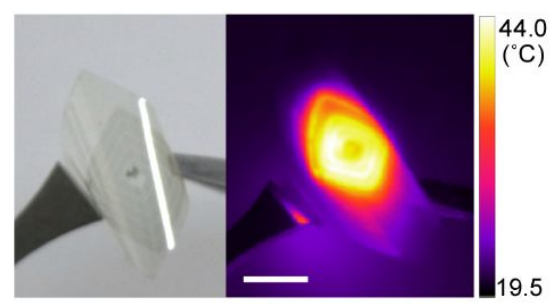

Figure 2. Transparent antenna and heater (a) Schematic illustration of transparent antenna. (b) Optical transmittance spectrum of the CuZr NTs-AgNWs hybrid antenna. (c) Resonance characteristics of the $\mathrm{Cu}$ antenna and CuZr NTs-AgNWs hybrid antenna. (d) Reflection values of the $\mathrm{Cu}$ antenna and $\mathrm{CuZr}$ NTs-AgNWs hybrid antenna as a function of tensile strain. In the case of $\mathrm{Cu}$ antenna, as tensile strain increases above $10 \%$, its reflection becomes 0 due to cracking of $\mathrm{Cu}$. (e) Temperature as a function of time with DC Voltage. (f) The photograph and IR image of the CuZr NTs-AgNWs hybrid antenna under bending stress. Scale bar, $1 \mathrm{~cm}$. 
a
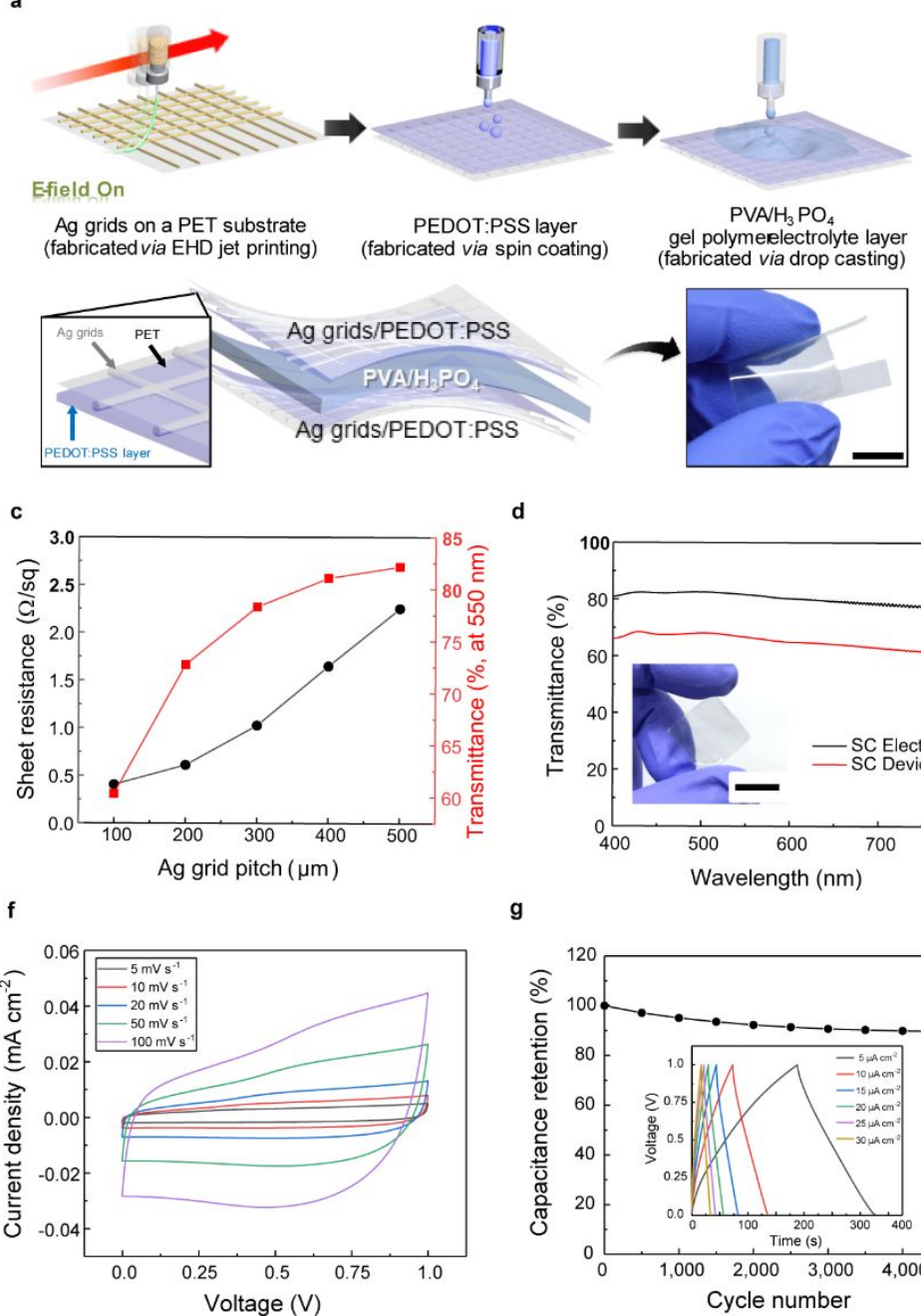
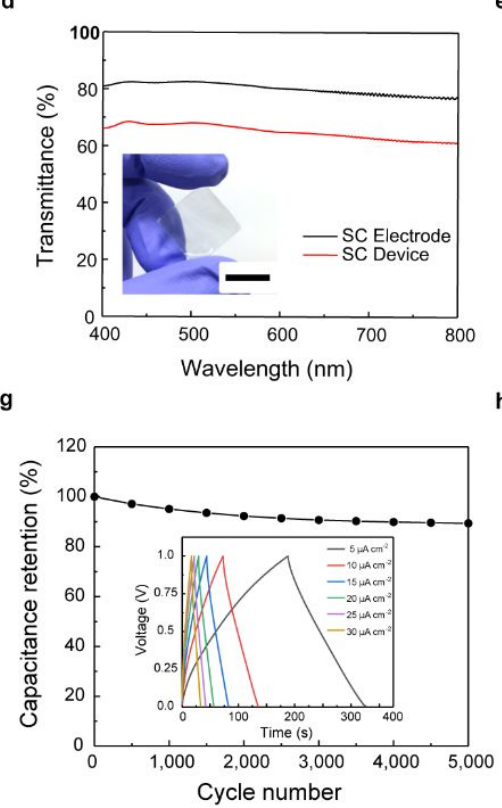

h
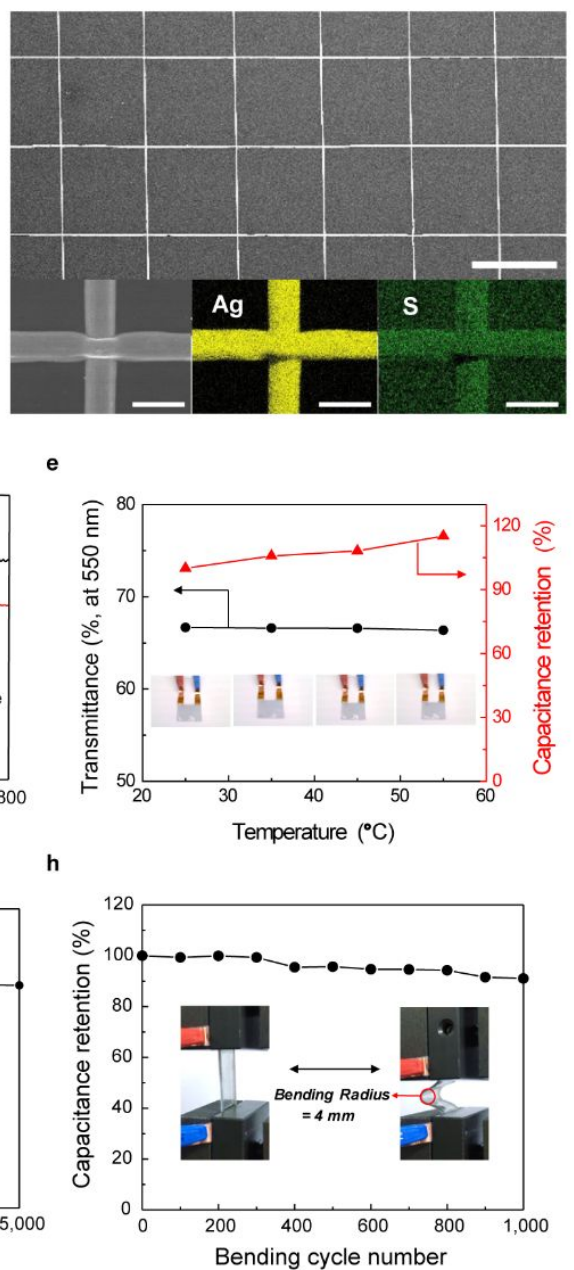

Figure 3. Fabrication and characterization of the transparent/flexible SC device. (a) Schematic of the overall fabrication process of the transparent SC device comprising on the EHD jetprinted Ag grids on a PET substrate, spin-coated PEDOT:PSS layer, and drop-casted $\mathrm{PVA} / \mathrm{H}_{3} \mathrm{PO}_{4}$ gel polymer electrolyte. Scale bar, $1 \mathrm{~cm}$. (b) SEM and EDS mapping images of the SC electrode (PEDOT:PSS electrode on top of the EHD jet-printed Ag grids). Scale bars, top: $1 \mathrm{~mm}$, bottoms: $20 \mu \mathrm{m}$. (c) Sheet electrical resistance and optical transmittance of the SC electrodes as a function of Ag grid pitch. (d) Transmittance profile (vs. wavelength) of the SC electrode, and SC device. Inset is a photograph of the SC device. Scale bar, $1 \mathrm{~cm}$. (e) Optical transmittance and capacitance retention of the $\mathrm{SC}$ device as a function of temperature. (f) CV curves of the SC device at various scan rates. (g) Cycling performance of the transparent SC (measured at a constant charge/discharge current density of $50 \mu \mathrm{A} \mathrm{cm}^{-2}$ ). Insets are the GCD profiles at various current densities of $5-30 \mu \mathrm{A} \mathrm{cm}^{-2}$. (h) Bending stability of the SC device with bending radii of $4 \mathrm{~mm}$ at a scan rate of $50 \mathrm{mV} \mathrm{s}^{-1}$. 

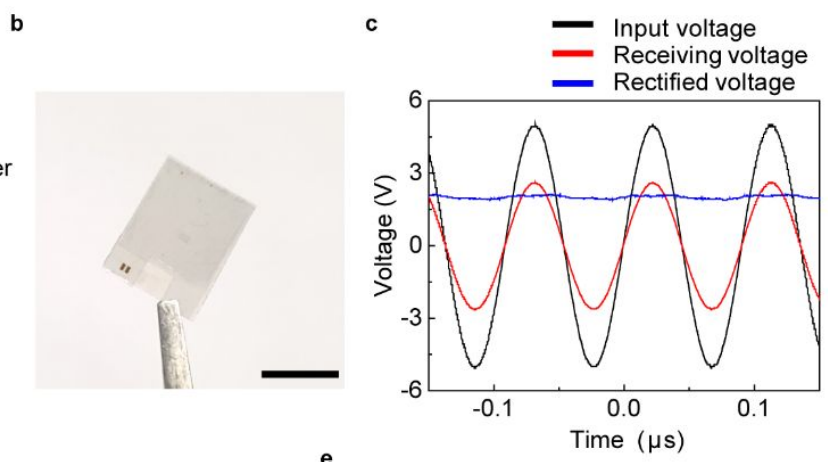

d
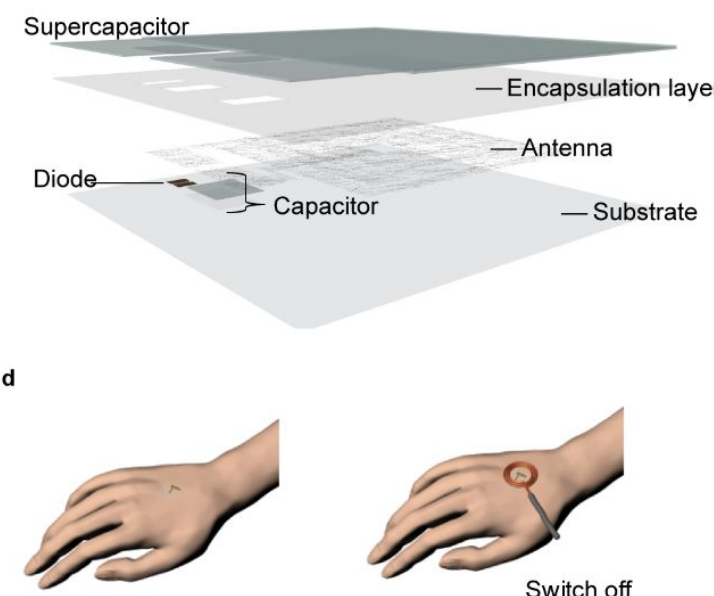

Switch off
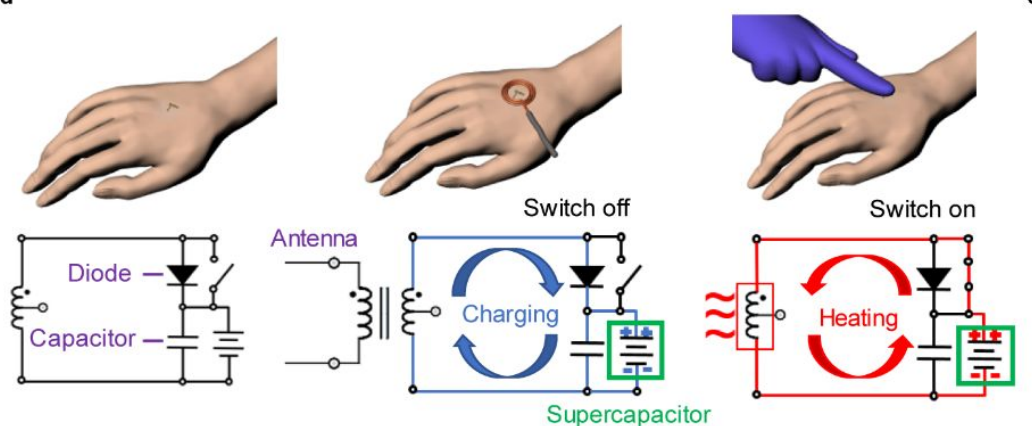

e
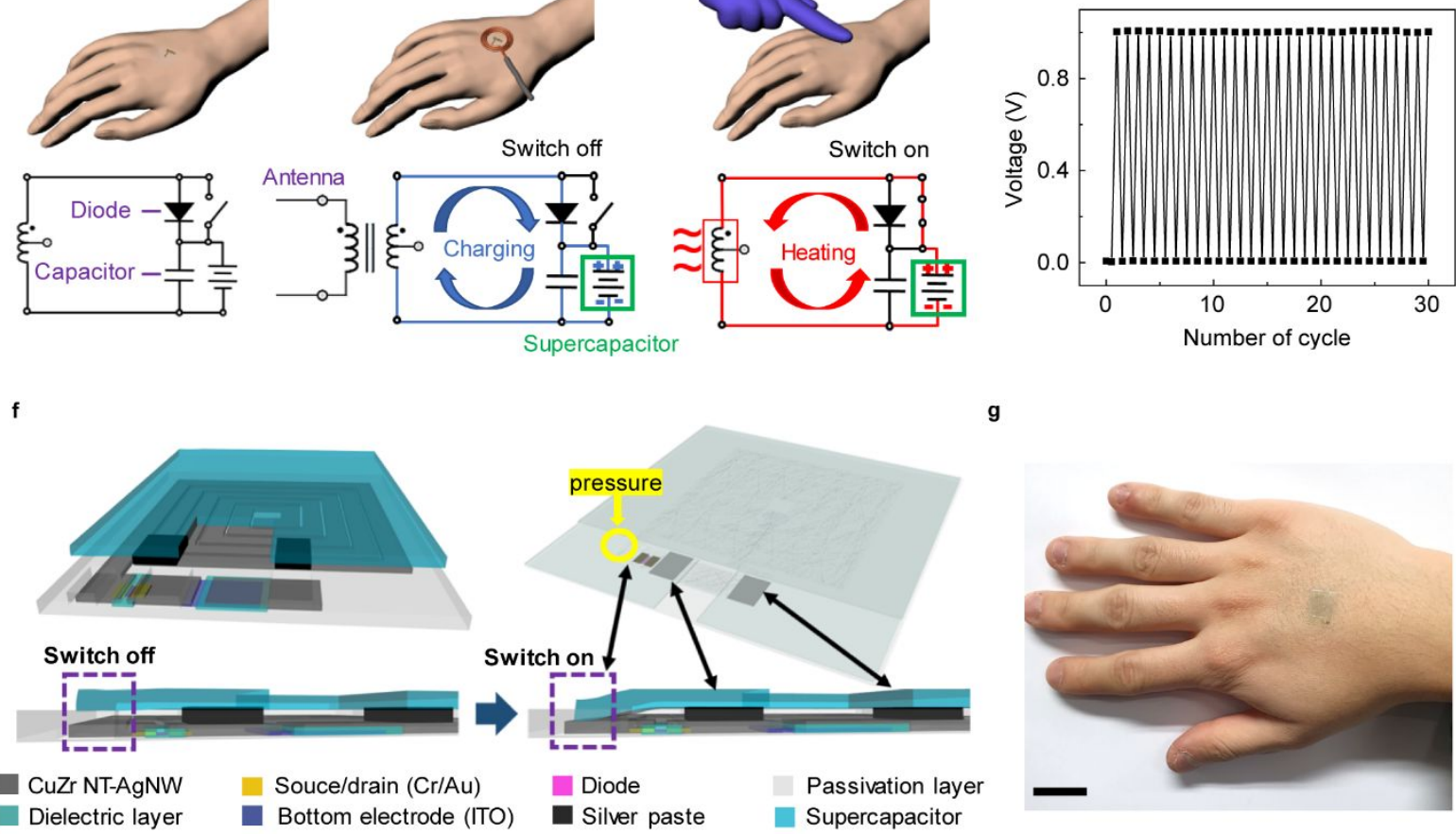

h
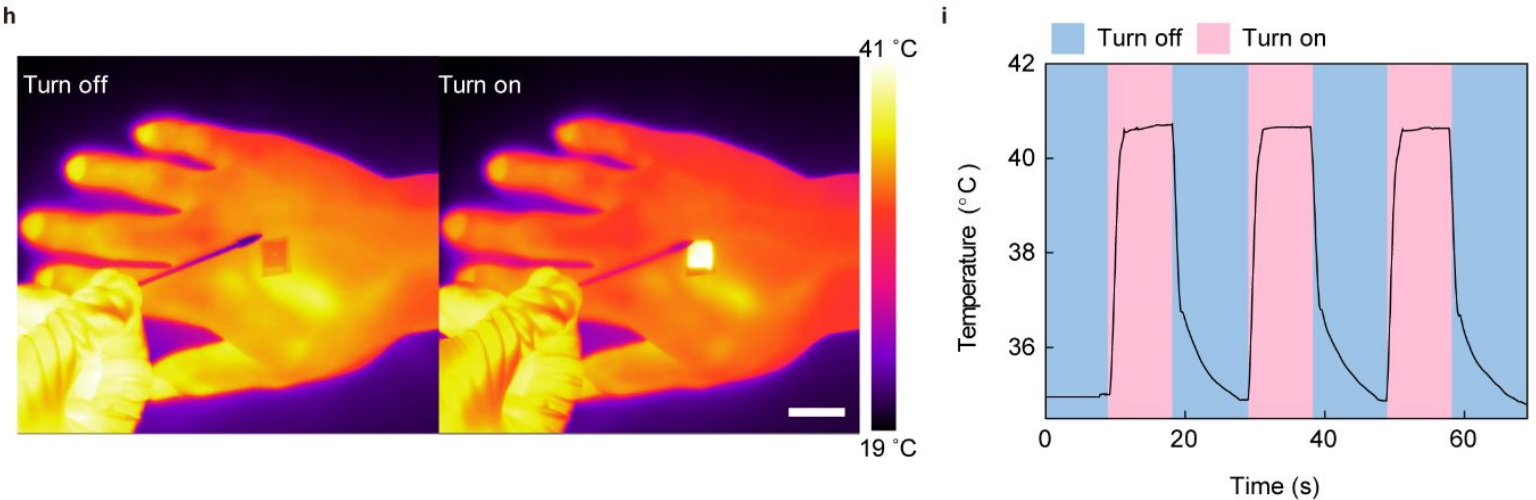

Figure 4. Thermal therapy patch (a) Schematic illustration of the integrated system, consisting of the rectifier (diode, capacitor), antenna, supercapacitor. (b) Photograph of wirelessly rechargeable, transparent and flexible heat patch. Scale bar, $1 \mathrm{~cm}$. (c) Rectified properties of the fabricated rectifier. (d) The changes in the circuit diagram of the heat patch following wireless charging and discharging. (e) Charging and discharging properties of the heat patch. (f) The operating mechanisms of the switch and the heat patch when external pressure is applied. (g) Photograph of the heat patch attached on the back of the hand. Scale bar, $2 \mathrm{~cm}$. (h) 
IR image of the heat patch with the switch turned on/off. Scale bars, $2 \mathrm{~cm}$. (i) Thermal stability and turn on/off test of heat patch with one charge. 
a
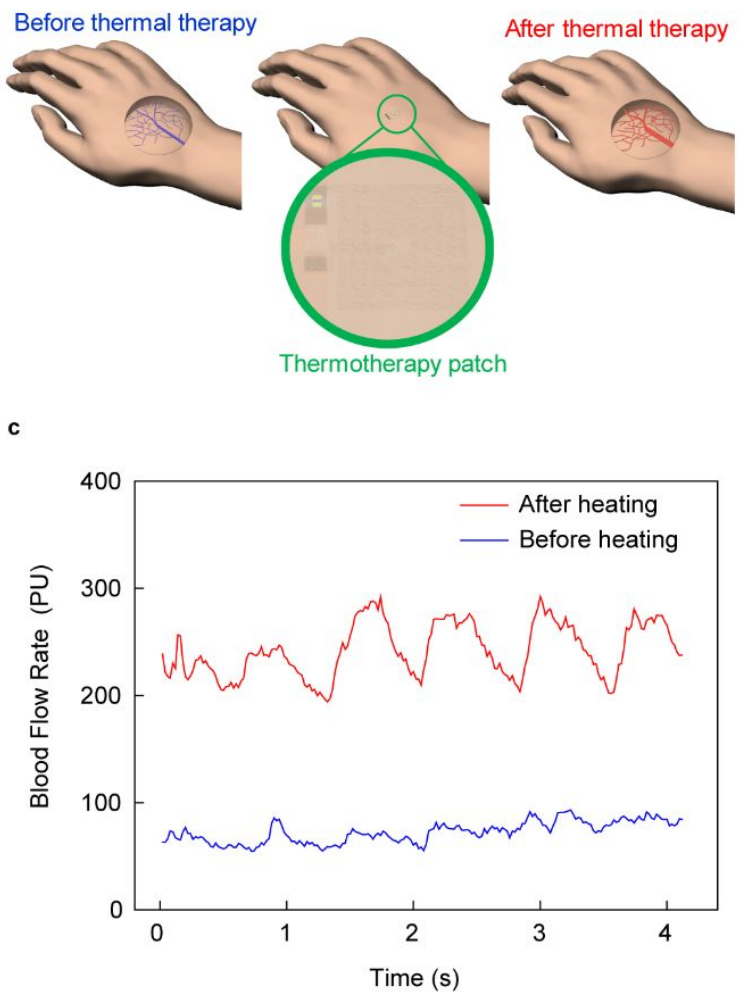
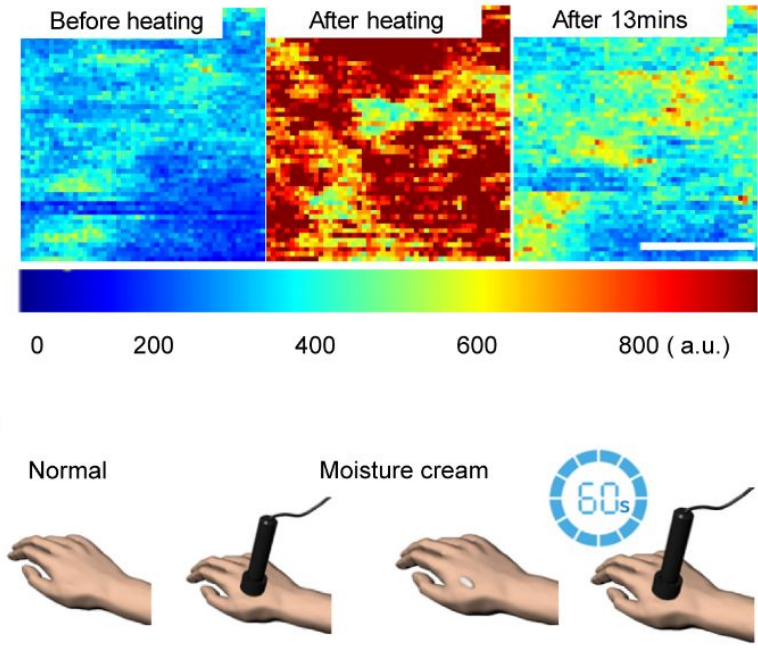

Moisture cream + thermal therapy $10,40,60 \mathrm{sec}$

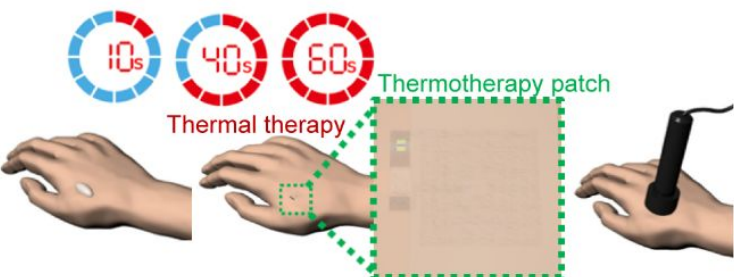

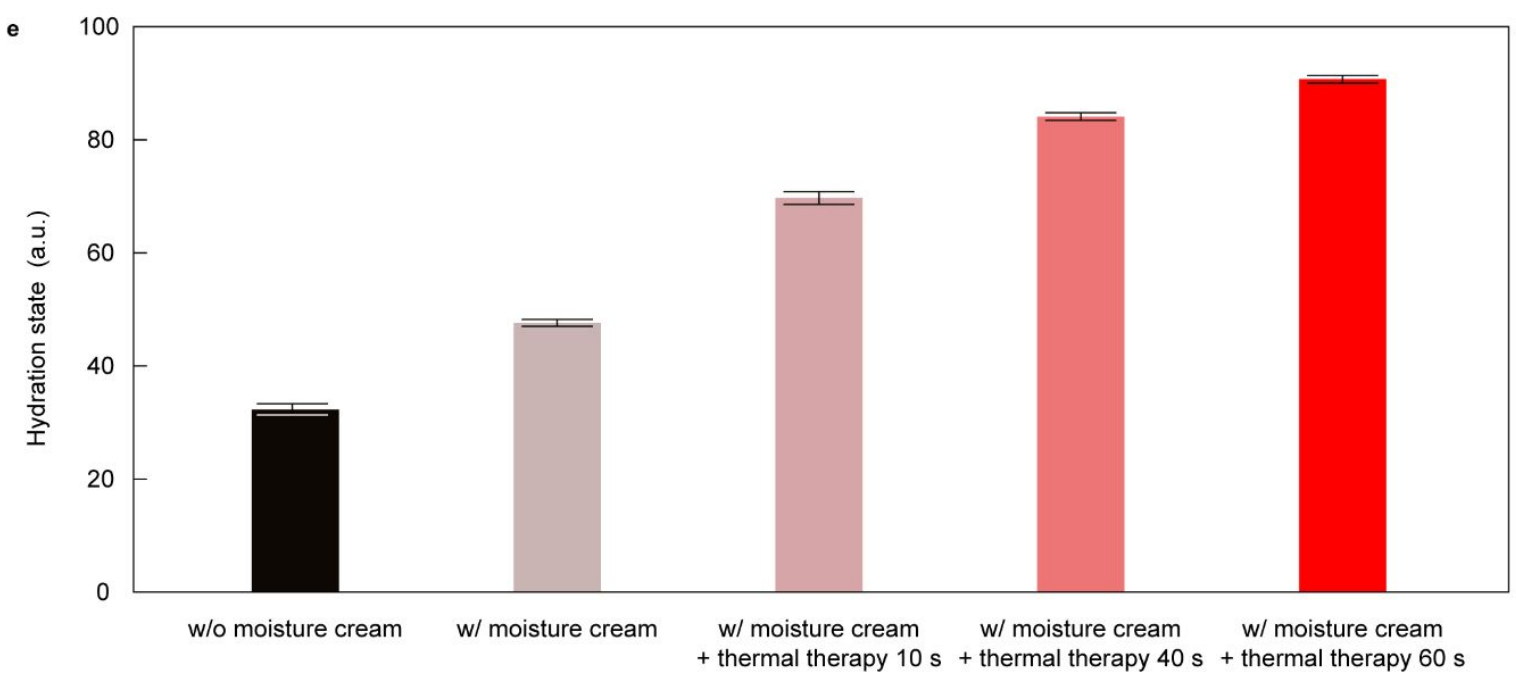

Figure 5. The effect of the thermal therapy (a) Schematic image showing blood flow rate change on the back of hand caused by thermal therapy. (b) Laser doppler images exhibiting blood flow rate changes with thermal therapy time. Scale bar, $5 \mathrm{~mm}$. (c) The results for laser doppler perfusion monitoring at one spot of skin. Measurements are expressed as perfusion units (PU). (d) A schematic image of hydration state measurements over time applied to thermal therapy. (e) Hydration state (a. u.) of the skin according to applied thermal therapy time immediately after applying moisture cream. The control group, the hydration state of the skin without moisture cream, were also compared. 


\section{ASSOCIATED CONTENT}

\section{Supporting Information}

The Supporting Information is available free of charge on the ACS Publications website at DOI: 10.1021/acs.nanolett.

- Methods and materials, comparison with previous studies, SEM images of CuZr NTs and hybrid electrode, photograph of $\mathrm{Cu}$ antenna, relative change in sheet resistance of the hybrid antenna and $\mathrm{Cu}$ antenna as a function of tensile stain, temperature changes as a function of the bending radius of the hybrid antenna, schematic of the EHD jetprinting equipment, SEM images of the EHD jet-printed Ag grid, optical properties of $\mathrm{SC}$ electrodes as a function of Ag grid pitch, cycling performance of the SC at $40{ }^{\circ} \mathrm{C}$, $\mathrm{CV}$ curves of the SC device as a function of bending deformation, photograph of antenna and rectifier circuit on polyimide (PI) substrate, fabrication procedures of the heat patch, optical transmittance of the heat patch in visible light range, characteristics of the Si diode, characteristics of the switch in the heat patch, details of laser Doppler images by thermal therapy time, details of moisture tests

- Supporting Movie 1. The fabrication of Ag grid current collector using the EHD jetprinting

- Supporting Movie 2. Wireless operation of the heat patch

\section{AUTHOR INFORMATION}

\section{Corresponding Author}

*E-mail: jang-ung@yonsei.ac.kr; syleek@unist.ac.kr; andrea.libassi@polimi.it 


\section{Author Contributions}

S.L., S.-W.K., M.G., H.S.A., and J.J. contributed equally to this work. S.L. and H.S.A. fabricated the device and conducted experiments for performance evaluation of the heat patches. S.-W.K. manufactured the transparent SCs. M.G. synthesized the CuZr NTs-AgNWs hybrid network. S.L., S.-W.K., M.G., and H.S.A. analyzed the data and wrote the manuscript. J.J. supported experiments for fabricating the device and contributed the revision of this manuscript. A.L.B. contributed to the analysis of the CuZr NTs-AgNWs hybrid network synthesis process and the revision of this manuscript. S.-Y.L. contributed to the appraisement of transparent SCs and the revision of this manuscript. J.-U.P. oversaw all research phases and revised the manuscript. All authors discussed and commented on the manuscript.

The authors declare no competing financial interest.

\section{ACKNOWLEDGMENT}

This work was supported by the Ministry of Science \& ICT (MSIT) and the Ministry of Trade, Industry and Energy (MOTIE) of Korea through the National Research Foundation (2019R1A2B5B03069358, 2016R1A5A1009926, and 2018R1A2A1A05019733), the Bio \& Medical Technology Development Program (2018M3A9F1021649), the Nano Material Technology Development Program (2015M3A7B4050308 and 2016M3A7B4910635), the Industrial Technology Innovation Program (10080577), and Industry Technology Development Program (10080540). Dr. Matteo Ghidelli would like to acknowledge the financial support of the Italian Embassy in Republic of Korea trough the "Borse di Viaggio" program enabling to develop a collaboration between Korean and Italian institutions. Also, the authors thank financial support by the Institute for Basic Science (IBS-R026-D1) and the Research Program (2018-22-0194) funded by Yonsei University. 


\section{REFERENCES}

(1) Bermúdez, G. S. C.; Karnaushenko, D. D.; Karnaushenko, D.; Lebanov, A.; Bischoff, L.; Kaltenbrunner, M.; Fassbender, J.; Schmidt, O. G.; Makarov, D. Magnetosensitive E-Skins with Directional Perception for Augmented Reality. Sci. Adv. 2018, 4 (1), eaao2623.

(2) Lee, H. E.; Lee, D.; Lee, T.-I.; Shin, J. H.; Choi, G.-M.; Kim, C.; Lee, S. H.; Lee, J. H.; Kim, Y. H.; Kang, S.-M.; et al. Wireless Powered Wearable Micro Light-Emitting Diodes. Nano Energy 2019, 55, 454-462.

(3) Sim, K.; Rao, Z.; Zou, Z.; Ershad, F.; Lei, J.; Thukral, A.; Chen, J.; Huang, Q.-A.; Xiao, J.; Yu, C. Metal Oxide Semiconductor Nanomembrane-Based Soft Unnoticeable Multifunctional Electronics for Wearable Human-Machine Interfaces. Sci. Adv. 2019, 5 (8), eaav9653.

(4) Jang, J.; Kim, H.; Ji, S.; Kim, H. J.; Kang, M. S.; Kim, T. S.; Won, J.; Lee, J.-H.; Cheon, J.; Kang, K.; Im, W. B.; Park, J.-U. Mechanoluminescent, Air-Dielectric $\mathrm{MoS}_{2}$ Transistors as Active-Matrix Pressure Sensors for Wide Detection Ranges from Footsteps to Cellular Motions. Nano Lett. 2020, 20 (1), 66-74.

(5) Liao, H.; Guo, X.; Wan, P.; Yu, G. Conductive MXene Nanocomposite Organohydrogel for Flexible, Healable, Low-Temperature Tolerant Strain Sensors. Adv. Funct. Mater. 2019, 29 (39), 1904507.

(6) Di, J.; Yao, S.; Ye, Y.; Cui, Z.; Yu, J.; Ghosh, T. K.; Zhu, Y.; Gu, Z. Stretch-Triggered Drug Delivery from Wearable Elastomer Films Containing Therapeutic Depots. $A C S$ Nano 2015, 9 (9), 9407-9415.

(7) Kim, J.; Kim, J.; Ku, M.; Cha, E.; Ju, S.; Park, W. Y.; Kim, K. H.; Kim, D. W.; Berggren, P.-O.; Park, J.-U. Intraocular Pressure Monitoring Following Islet Transplantation to the Anterior Chamber of the Eye. Nano Lett. 2020, 20 (3), 1517-1525. 
(8) Park, J.; Kim, J.; Kim, S.-Y.; Cheong, W. H.; Jang, J.; Park, Y.-G.; Na, K.; Kim, Y.-T.; Heo, J. H.; Lee, C. Y.; et al. Soft, Smart Contact Lenses with Integrations of Wireless Circuits, Glucose Sensors, and Displays. Sci. Adv. 2018, 4 (1), eaap9841.

(9) Kim, S. B.; Koo, J.; Yoon, J.; Hourlier-Fargette, A.; Lee, B.; Chen, S.; Jo, S.; Choi, J.; Oh, Y. S.; Lee, G.; et al. Soft, Skin-Interfaced Microfluidic Systems with Integrated Enzymatic Assays for Measuring the Concentration of Ammonia and Ethanol in Sweat. Lab Chip 2020, 20 (1), 84-92.

(10) An, B. W.; Shin, J. H.; Kim, S.-Y.; Kim, J.; Ji, S.; Park, J.; Lee, Y.; Jang, J.; Park, Y.G.; Cho, E.; et al. Smart Sensor Systems for Wearable Electronic Devices. Polymers 2017, $9(8), 303$.

(11) Bandodkar, A. J.; Gutruf, P.; Choi, J.; Lee, K.; Sekine, Y.; Reeder, J. T.; Jeang, W. J.; Aranyosi, A. J.; Lee, S. P.; Model, J. B.; et al. Battery-Free, Skin-Interfaced Microfluidic/Electronic Systems for Simultaneous Electrochemical, Colorimetric, and Volumetric Analysis of Sweat. Sci. Adv. 2019, 5 (1), eaav3294.

(12) Lee, H.; Song, C.; Hong, Y. S.; Kim, M. S.; Cho, H. R.; Kang, T.; Shin, K.; Choi, S. H.; Hyeon, T.; Kim, D.-H. Wearable/Disposable Sweat-Based Glucose Monitoring Device with Multistage Transdermal Drug Delivery Module. Sci. Adv. 2017, 3 (3), e1601314.

(13) Choi, S.; Park, J.; Hyun, W.; Kim, J.; Kim, J.; Lee, Y. B.; Song, C.; Hwang, H. J.; Kim, J. H.; Hyeon, T.; et al. Stretchable Heater Using Ligand-Exchanged Silver Nanowire Nanocomposite for Wearable Articular Thermotherapy. ACS Nano 2015, 9 (6), 66266633.

(14) Jeong, Y. R.; Lee, G.; Park, H.; Ha, J. S. Stretchable, Skin-Attachable Electronics with Integrated Energy Storage Devices for Biosignal Monitoring. Acc. Chem. Res. 2019, 52 (1), 91-99.

(15) Jeong, H.; Wang, L.; Ha, T.; Mitbander, R.; Yang, X.; Dai, Z.; Qiao, S.; Shen, L.; Sun, 
N.; Lu, N. Modular and Reconfigurable Wireless E-Tattoos for Personalized Sensing. Adv. Mater. Technol. 2019, 4 (8), 1900117.

(16) Stier, A.; Halekote, E.; Mark, A.; Qiao, S.; Yang, S.; Diller, K.; Lu, N. Stretchable Tattoo-Like Heater with On-Site Temperature Feedback Control. Micromachines 2018, $9(4), 170$.

(17) Son, D.; Kang, J.; Vardoulis, O.; Kim, Y.; Matsuhisa, N.; Oh, J. Y.; To, J. W.; Mun, J.; Katsumata, T.; Liu, Y.; et al. An Integrated Self-Healable Electronic Skin System Fabricated via Dynamic Reconstruction of a Nanostructured Conducting Network. Nature Nanotechnol. 2018, 13 (11), 1057-1065.

(18) Lee, Y.; Cha, S. H.; Kim, Y.-W.; Choi, D.; Sun, J.-Y. Transparent and Attachable Ionic Communicators Based on Self-Cleanable Triboelectric Nanogenerators. Nat. Commun. 2018, 9 (1), 1-8.

(19) An, B. W.; Gwak, E.-J.; Kim, K.; Kim, Y.-C.; Jang, J.; Kim, J.-Y.; Park, J.-U. Stretchable, Transparent Electrodes as Wearable Heaters Using Nanotrough Networks of Metallic Glasses with Superior Mechanical Properties and Thermal Stability. Nano Lett. 2016, 16 (1), 471-478.

(20) Jang, J.; Hyun, B. G.; Ji, S.; Cho, E.; An, B. W.; Cheong, W. H.; Park, J.-U. Rapid Production of Large-Area, Transparent and Stretchable Electrodes Using Metal Nanofibers as Wirelessly Operated Wearable Heaters. NPG Asia Mater. 2017, 9 (9), e432.

(21) Ji, S.; Park, J.; Jo, Y.; Kim, Y.-B.; Jang, J.; Kim, S.-K.; Jeong, S.; Park, J.-U. Haze-Free Transparent Electrodes Using Metal Nanofibers with Carbon Shells for HighTemperature Stability. Applied Surface Science 2019, 483, 1101-1109.

(22) Wu, H.; Kong, D.; Ruan, Z.; Hsu, P.-C.; Wang, S.; Yu, Z.; Carney, T. J.; Hu, L.; Fan, S.; Cui, Y. A Transparent Electrode Based on a Metal Nanotrough Network. Nature 
Nanotechnol. 2013, 8 (6), 421-425.

(23) Liu, Y.; Zhang, J.; Gao, H.; Wang, Y.; Liu, Q.; Huang, S.; Guo, C. F.; Ren, Z. CapillaryForce-Induced Cold Welding in Silver-Nanowire-Based Flexible Transparent Electrodes. Nano Lett. 2017, 17 (2), 1090-1096.

(24) Lee, M.-S.; Lee, K.; Kim, S.-Y.; Lee, H.; Park, J.; Choi, K.-H.; Kim, H.-K.; Kim, D.-G.; Lee, D.-Y.; Nam, S.; et al. High-Performance, Transparent, and Stretchable Electrodes Using Graphene-Metal Nanowire Hybrid Structures. Nano Lett. 2013, 13 (6), 28142821.

(25) An, B. W.; Hyun, B. G.; Kim, S.-Y.; Kim, M.; Lee, M.-S.; Lee, K.; Koo, J. B.; Chu, H. Y.; Bae, B.-S.; Park, J.-U. Stretchable and Transparent Electrodes Using Hybrid Structures of Graphene-Metal Nanotrough Networks with High Performances and Ultimate Uniformity. Nano Lett. 2014, 14 (11), 6322-6328.

(26) Im, H.-G.; An, B. W.; Jin, J.; Jang, J.; Park, Y.-G.; Park, J.-U.; Bae, B.-S. A HighPerformance, Flexible and Robust Metal Nanotrough-Embedded Transparent Conducting Film for Wearable Touch Screen Panels. Nanoscale 2016, 8 (7), 3916-3922.

(27) Eason, R., Ed.; Pulsed Laser Deposition of Thin Films: Applications-Led Growth of Functional Materials. Wiley-Interscience: Hoboken, N.J, 2007.

(28) Ghidelli, M.; Gravier, S.; Blandin, J.-J.; Djemia, P.; Mompiou, F.; Abadias, G.; Raskin, J.-P.; Pardoen, T. Extrinsic Mechanical Size Effects in Thin ZrNi Metallic Glass Films. Acta Materialia 2015, 90, 232-241.

(29) Ghidelli, M.; Idrissi, H.; Gravier, S.; Blandin, J.-J.; Raskin, J.-P.; Schryvers, D.; Pardoen, T. Homogeneous Flow and Size Dependent Mechanical Behavior in Highly Ductile Zr65Ni35 Metallic Glass Films. Acta Materialia 2017, 131, 246-259.

(30) Chu, J. P.; Jang, J. S. C.; Huang, J. C.; Chou, H. S.; Yang, Y.; Ye, J. C.; Wang, Y. C.; Lee, J. W.; Liu, F. X.; Liaw, P. K.; et al. Thin Film Metallic Glasses: Unique Properties 
and Potential Applications. Thin Solid Films 2012, 520 (16), 5097-5122.

(31) Etiemble, A.; Der Loughian, C.; Apreutesei, M.; Langlois, C.; Cardinal, S.; Pelletier, J. M.; Pierson, J.-F.; Steyer, P. Innovative Zr-Cu-Ag Thin Film Metallic Glass Deposed by Magnetron PVD Sputtering for Antibacterial Applications. Journal of Alloys and Compounds 2017, 707, 155-161.

(32) Ivanisenko, Y.; Kübel, C.; Nandam, S. H.; Wang, C.; Mu, X.; Adjaoud, O.; Albe, K.; Hahn, H. Structure and Properties of Nanoglasses. Adv. Eng. Mater. 2018, 20 (12), 1800404.

(33) Jang, Y.; Kim, J.; Byun, D. Invisible Metal-Grid Transparent Electrode Prepared by Electrohydrodynamic (EHD) Jet Printing. J. Phys. D: Appl. Phys. 2013, 46 (15), 155103.

(34) Yang, S. M.; Lee, Y. S.; Jang, Y.; Byun, D.; Choa, S.-H. Electromechanical Reliability of a Flexible Metal-Grid Transparent Electrode Prepared by Electrohydrodynamic (EHD) Jet Printing. Microelectronics Reliability 2016, 65, 151-159.

(35) Cheng, T.; Zhang, Y.-Z.; Zhang, J.-D.; Lai, W.-Y.; Huang, W. High-Performance FreeStanding PEDOT:PSS Electrodes for Flexible and Transparent All-Solid-State Supercapacitors. Journal of Materials Chemistry A 2016, 4 (27), 10493-10499.

(36) Li, Z.; Ma, G.; Ge, R.; Qin, F.; Dong, X.; Meng, W.; Liu, T.; Tong, J.; Jiang, F.; Zhou, Y.; et al. Free-Standing Conducting Polymer Films for High-Performance Energy Devices. Angewandte Chemie International Edition 2016, 55 (3), 979-982.

(37) Cai, G.; Darmawan, P.; Cui, M.; Wang, J.; Chen, J.; Magdassi, S.; Lee, P. S. Highly Stable Transparent Conductive Silver Grid/PEDOT:PSS Electrodes for Integrated Bifunctional Flexible Electrochromic Supercapacitors. Adv. Energy Mater 2016, 6 (4), 1501882.

(38) Lee, Y.; Jin, W.-Y.; Young Cho, K.; Kang, J.-W.; Kim, J. Thermal Pressing of a MetalGrid Transparent Electrode into a Plastic Substrate for Flexible Electronic Devices. 
Journal of Materials Chemistry C 2016, 4 (32), 7577-7583.

(39) Park, J.; Ahn, D. B.; Kim, J.; Cha, E.; Bae, B.-S.; Lee, S.-Y.; Park, J.-U. Printing of Wirelessly Rechargeable Solid-State Supercapacitors for Soft, Smart Contact Lenses with Continuous Operations. Sci. Adv. 2019, 5 (12), eaay0764.

(40) Lehmann, J. F.; Warren, C. G.; Scham, S. M. Therapeutic Heat and Cold. Clinical Orthopaedics and Related Research ${ }^{\circledR}$ 1974, 99, 207.

(41) Bayata, S.; Türel Ermertcan, A. Thermotherapy in Dermatology. Cutaneous and Ocular Toxicology 2012, 31 (3), 235-240.

(42) Dilek, B.; Gözüm, M.; Şahin, E.; Baydar, M.; Ergör, G.; El, Ö.; Bircan, Ç.; Gülbahar, S. Efficacy of Paraffin Bath Therapy in Hand Osteoarthritis: A Single-Blinded Randomized Controlled Trial. Archives of Physical Medicine and Rehabilitation 2013, $94(4), 642-649$.

(43) Briers, J. D. Laser Doppler, Speckle and Related Techniques for Blood Perfusion Mapping and Imaging. Physiol. Meas. 2001, 22 (4), R35-R66.

(44) Fredriksson, I.; Larsson, M.; Strömberg, T. Measurement Depth and Volume in Laser Doppler Flowmetry. Microvascular Research 2009, 78 (1), 4-13. 
For Table of Contents Only

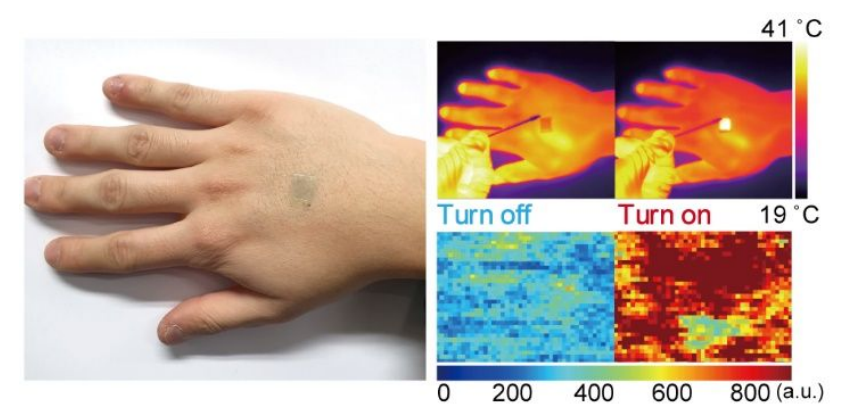

